\title{
BMJ Open Safety and feasibility of using acellular sterile filtered amniotic fluid as a treatment for patients with COVID-19: protocol for a randomised, double- blinded, placebo-controlled clinical trial
}

\author{
Joseph E Tonna (D) , ${ }^{1}$ Jan Pierce, ${ }^{2}$ Nathan Hatton, ${ }^{3}$ Giavonni Lewis, ${ }^{1}$ \\ John D Phillips, ${ }^{3}$ Alyssa Messina, ${ }^{1}$ Chloe R Skidmore, ${ }^{1}$ Kirsten Taylor, ${ }^{1}$ \\ Craig H Selzman ${ }^{1}$
}

To cite: Tonna JE, Pierce J, Hatton N, et al. Safety and feasibility of using acellular sterile filtered amniotic fluid as a treatment for patients with COVID-19: protocol for a randomised, doubleblinded, placebo-controlled clinical trial. BMJ Open 2021;11:e045162. doi:10.1136/ bmjopen-2020-045162

- Prepublication history and supplemental material for this paper is available online. To view these files, please visit the journal online (http://dx.doi org/10.1136/bmjopen-2020045162).

Received 25 September 2020 Revised 12 January 2021 Accepted 21 January 2021

D) Check for updates

(c) Author(s) (or their employer(s)) 2021. Re-use permitted under CC BY-NC. No commercial re-use. See rights and permissions. Published by BMJ.

${ }^{1}$ Department of Surgery, University of Utah Health, Salt Lake City, Utah, USA

${ }^{2}$ CellReGen, University of Utah Health, Salt Lake City, Utah, USA ${ }^{3}$ Department of Medicine, University of Utah Health, Salt Lake City, Utah, USA

Correspondence to Dr Joseph E Tonna; joseph.tonna@hsc.utah.edu

\section{ABSTRACT}

Introduction Human amniotic fluid (hAF) has been shown to reduce inflammation in multiple experimental models. hAF has previously been approved by the US Food and Drug Administration (FDA) as a human cellular and tissue product for tissue injury for human administration, and used safely in thousands of patients as a therapeutic treatment for diverse conditions. Given the profound inflammatory response observed in patients with COVID-19, and the successful completion of 10-patient pilot study of intravenous hAF, we present a trial design for a larger clinical trial of intravenous hAF for the treatment of COVID-19.

Methods and analysis This paper describes the methodology of a phase $1 / /$ randomised, double-blinded, placebo-controlled clinical trial to determine the safety and feasibility of using acellular sterile filtered amniotic fluid as a treatment for patients with COVID-19. Primary outcome will be the change in C-reactive protein. Secondary outcomes include safety, biomarker inflammatory levels and clinically relevant outcomes at 30 days, including mortality, ventilator-free days and hospital and intensive care unit length of stay. Exploratory outcomes of healthrelated quality-of-life patient-reported outcomes will be collected. Hospitalised patients with laboratory-confirmed COVID-19 will be recruited.

Ethics and dissemination This study was approved by the University of Utah Institutional Review Board (IRB_0013292), approved by the US FDA under Investigational New Drug (No 23369) and is registered on ClinicalTrials.gov. Results will be disseminated via peerreviewed publications and conference presentations. Trial registration number NCT04497389; Pre-results.

\section{INTRODUCTION}

Human amniotic fluid (hAF) and human amniotic membrane have been shown to reduce inflammation, ${ }^{1-3}$ have antimicrobial properties $^{4-6}$ and have a low risk of immunogenicity. ${ }^{7} \mathrm{hAF}$ is naturally sterile, but can be further prepared as a filtered fluid and
Strengths and limitations of this study

- A protocol for a double-blinded, placebo-controlled, randomised clinical trial in patients with COVID-19.

- The study will demonstrate the effect of purified amniotic fluid, a naturally derived anti-inflammatory, as a novel intravenous therapeutic on the inflammatory response of COVID-19.

- Trial inclusion criteria are broad, allowing for enrolment of hospitalised patients with COVID-19 with few restrictions.

- Limited by the sample size of 60 patients.

- Sample size is sufficient for safety and biochemical outcomes, but may not be sufficient to show an effect on clinical outcomes such as mortality.

meets United States Pharmacopeia (USP) $<71>$ sterility guidelines for delivery as a therapeutic treatment for a variety of conditions. In this capacity, purified hAF is devoid of any cellular products (not to be confused with umbilical cord-derived, AF-derived stem cell products, or AF embolism), and is a nonantigenic solution of $>1000$ proteins. Furthermore, hAF has previously been approved by the US Food and Drug Administration (FDA) as a human cellular and tissue product $(\mathrm{HCT} / \mathrm{P})$ under 21 Code of Federal Regulations (CFR) $1271^{8}$ for tissue injury, allowing it to be administered without an Investigational New Drug (IND) application; however, new FDA guidance requires that hAF trial have an IND number or exemption to comply with current regulations. To date, hAF has been used safely in thousands of patients as a therapeutic treatment for diverse conditions, including intractable epithelial defects, burns, diabetic/peripheral vascular ulcers, partial limbal cell deficiencies, peripheral 
nerve regeneration, tendon repair and Stevens-Johnson syndrome. ${ }^{9-13}$

Patients with COVID-19 have a large inflammatory response, with marked elevation of proinflammatory cytokines C-reactive protein (CRP), interleukin (IL)-1 $\beta$ and IL-6. ${ }^{14-17}$ This presents a potential role for hAF, given its successful use in other conditions to mitigate inflammation and decrease fibrosis. Recently, our group successfully completed a pilot study of intravenous hAF as a treatment for the inflammation associated with COVID-19 in 10 patients. Based on these pilot results, we present a study protocol for a randomised, double-blinded, placebocontrolled trial of intravenous, acellular, sterile filtered hAF for the treatment of moderate COVID-19. Merging the COVID-19-related observations and our experience with $\mathrm{hAF}$, we hypothesise that systemic administration of hAF may assist in the treatment of this disease, specifically by reducing inflammation. Ideally, this treatment could impact disease progression, decrease the number of infected patients requiring critical care, thereby impacting patient outcomes as well as the socioeconomic burden of this pandemic on precious resources.

\section{MATERIALS AND METHODS}

\section{Overview of study design}

The trial began enrolment on 28 October 2020 as a single-centre, prospective, randomised, double-blinded, placebo-controlled trial. We may enrol additional sites as the trial proceeds. Participants are recruited from hospitalised inpatients with COVID-19. Figure 1 shows the study screening and enrolment flow chart. Trial target enrolment is 60 patients. This study protocol follows the Standard Protocol Items: Recommendations for Interventional Trials, version $2013{ }^{18}$ Our hypothesis is that daily intravenous administration of acellular sterile filtered hAF in hospitalised patients with symptomatic COVID-19 will decrease inflammatory biomarker CRP compared with placebo. Estimated study completion date is 15 September 2021.

\section{Participant selection and recruiting process}

We are screening hospitalised patients with confirmed COVID-19 infection among hospitalised patients. Ideal subjects are mild to moderately, but not severely, ill with COVID-19. Screening includes an initial electronic medical record review by study coordinators at each site to identify patients with laboratory-confirmed COVID-19. Patient notes will be screened for evidence of active symptoms as defined in inclusion criteria. Given the contagiousness of COVID-19, eligible patients, or their legally authorised representatives (LAR), will be contacted via phone for e-consent.

\section{Inclusion criteria}

Patients are eligible for trial enrolment if they fulfil all the criteria:

- At least 18 years of age.

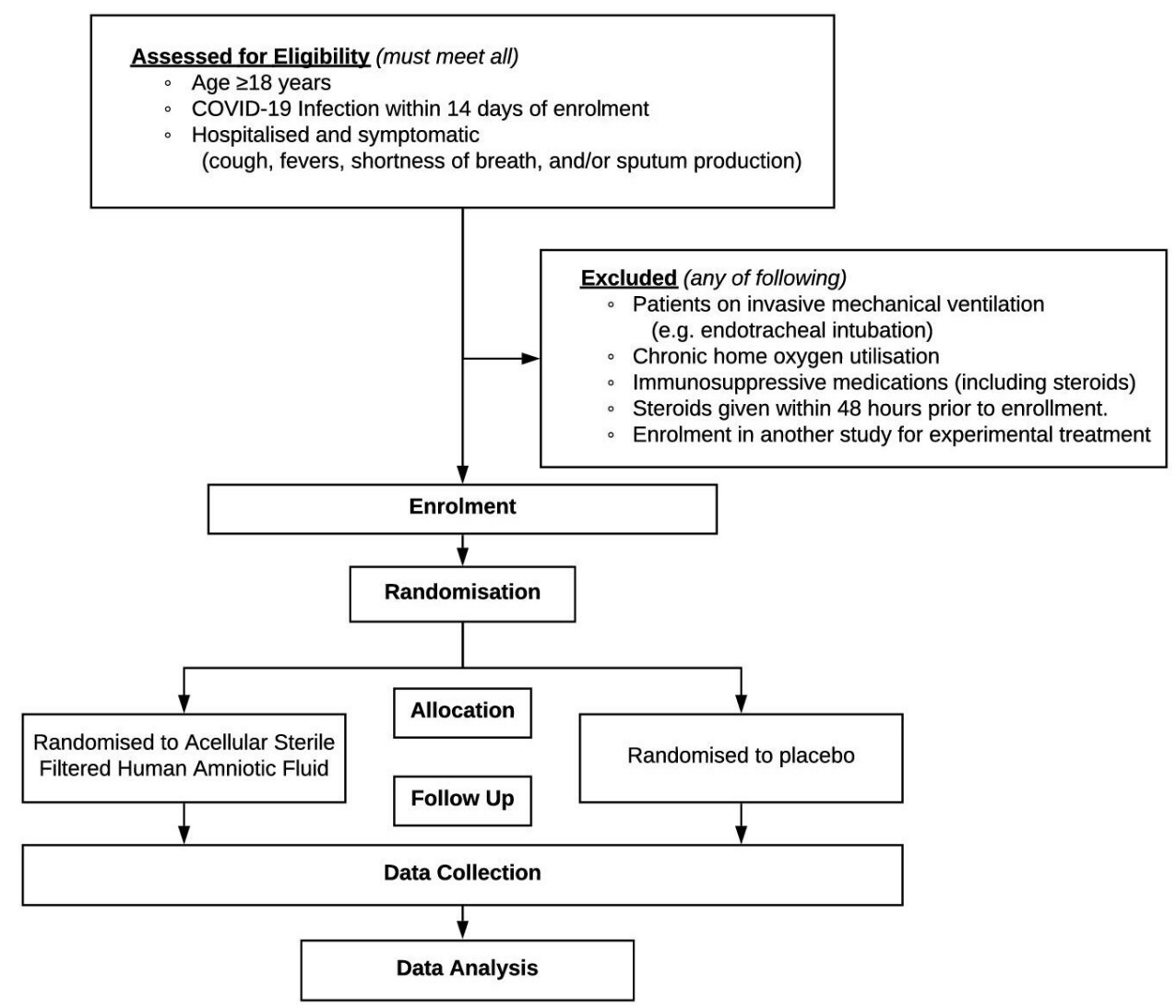

Figure 1 Patient screening and enrolment flow chart. 
- SARS-CoV-2 laboratory positive test, obtained within 14 days of enrolment.

- Hospitalised and symptomatic (cough, fevers, shortness of breath and/or sputum production).

- Has a room air pulse oximetry of $\leq 94 \%$ and requires supplemental oxygen therapy.

- Patients of childbearing potential who agree to use acceptable methods of contraception for 90 days after last administration of study investigational product (IP).

- Patients who are receiving standard of care therapies for COVID-19 that are not FDA approved are eligible for this study.

- Patients must be able to consent to the study (eg, Glasgow Coma Scale score $\geq 14$ ).

- Patients are required to have controlled blood pressure $<160 / 96$ and have a pulse $<110$.

\section{Exclusion criteria}

Patients will be excluded if they fulfil any of the exclusion criteria:

- Patients on invasive mechanical ventilation (eg, endotracheal intubation).

- Patients on non-invasive positive pressure ventilation.

- Patients on $>12 \mathrm{~L} / \mathrm{min}$ via non-rebreather or $>80 \%$ oxygen via high flow nasal cannula.

- Patients who, in the opinion of the investigator, have impending respiratory failure, defined as requiring rapidly escalating oxygen supplementation.

- Chronic home oxygen utilisation.

- Home use of immunosuppressive medications (including steroids).

- Women who are pregnant, breast feeding or become pregnant during the study.

- Patients with a haemoglobin $<90 \mathrm{~g} / \mathrm{L}$.

- Patients diagnosed with stage 4 or 5 chronic kidney disease.

- Patients diagnosed with class 3 or 4 congestive heart failure.

- Patients with a left ventricular assist device.

- Patients with thromboembolic phenomena.

- Patients with type 2 and above heart block.

- Patients with established positive bacterial blood cultures prior to enrolment.

- Patients with ongoing pericardial effusion, or ascites.

- Patients with clinically significant arrhythmia.

- Patients with liver function tests (alanine aminotransferase or aspartate aminotransferase) $>3 \times$ normal.

- Patients with untreated HIV infection.

- Patients diagnosed with end-stage organ disease.

\section{Ethics and informed consent}

This trial was approved by the University of Utah Institutional Review Board (IRB_0013292), received approval from the US FDA for an IND (No 23369) and is registered on ClinicalTrials.gov. The trial registration adheres to all items from the WHO Trial Registration Data Set. The study protocol (Version: 10 August 2020) is listed in the online supplemental file 1 . Written informed consent is obtained by study staff from each enrolled patient. The consent form is available in the online supplemental file 1 and includes a discussion of the risks and benefits of participation, and the ability to withdraw at any time without consequence.

Given the contagiousness of COVID-19, the consent process will happen remotely. A link to Research Electronic Data Capture (REDCap) will be sent via electronic mail (email) to the patient. The patient will be asked to access it via their personal device to minimise contact between patients and research staff. An outline of the study will be presented, and the participant will be free to ask questions and carefully consider whether or not to participate. They will receive a copy of their signed consent form via email. In cases where patients are unable to consent (eg, they are sedated), a LAR will be contacted for consent. If and when patients recover decision-making capacity, they will be fully reconsented. Research staff will write a consent note for all consents.

Any protocol amendments will be submitted to the IRB for approval prior to change. Consents will be updated as required. The protocol will then be updated on ClinicalTrials.gov.

\section{Funding source}

The trial is funded by the University of Utah through an Immunology, Inflammation and Infectious Disease (3i) Initiative/Vice-President for Research grant (https:// research.utah.edu). It is additionally funded by the State of Utah CARES Act. The study sponsors had no role in the design of the study, and will have no role in the conduct of the study or the interpretation of the results.

\section{Baseline assessments}

Baseline assessments are listed in box 1 and include patient demographics, baseline clinical and disease

\section{Box 1 Baseline demographic, clinical and laboratory} assessments obtained after enrolment but prior to randomisation

- Demographics (age, sex, height, weight, race/ethnicity, study hospital admission date and time).

- Medical history.

- Date of symptom onset.

- Date of confirmed positive COVID-19 test.

- Vital signs (heart rate, blood pressure, oxygen saturation, respiratory rate, temperature).

- Glasgow Coma Scale.

- Respiratory support modality and settings.

- Arterial blood gas.

- Complete blood count with autodifferential.

- Complete metabolic panel.

- Blood glucose (for patients with diabetes).

- Inflammatory biomarkers (interleukin (IL)-6, C-reactive protein (CRP), D-dimer, lactate dehydrogenase). 
characteristics, markers of severity of illness and inflammatory markers.

\section{Randomisation and concealment}

On enrolment, participants will be assigned a unique study participant code based on the unique combination of their name, medical record number, hospital encounter number and date of birth. The key to link this study participant code with the participant identity will be stored on a dual authentication, password-secured, Health Insurance Portability and Accountability Act (HIPAA)-compliant website of an unblinded study staff member. After assignment of the study code to the participant, all subsequent data entry will be identified by the study participant code, without the use of the patient's name, medical record number, hospital encounter number or date of birth. The participant's name, medical record number, hospital encounter number and date of birth will not be stored along with their data.

Patients will be randomised 1:1 to intervention versus control using a web-based interactive response system (REDCap randomisation module). Randomisation will occur using random permuted blocks of 2-4. When a patient has been enrolled, the study coordinator will $\log$ into the web-based REDCap interface and enter the patient. The study coordinator will then click on the randomisation button and a randomisation number will be assigned. The University of Utah Cell Therapy and Regenerative Medicine (CellReGen) will receive the subject ID and the assigned randomisation number with corresponding allocation (intervention (hAF) vs placebo). CellReGen will then prepare the study drug according to standard of care for clinical drug preparation. The therapy will be prepared as a sterile fluid for injection, labelled with the patient's name, medical record number and date and time, along with a 'study drug' label, per standard practice for clinical drug preparation. The study drug will then be delivered to the patient's nurse for administration.

\section{Amniotic fluid}

hAF for the trial is purchased from CellReGen. CellReGen has a proprietary process to collect $\mathrm{AF}$ at the time of caesarean section delivery. As such, there is no hazard to the baby. Once fluid is obtained, it is purified and decellularised through a proprietary filtration process with the end filter being a $0.2 \mu \mathrm{m}$ filter. This has been verified through particulate analysis and microscopic evaluation.

\section{Intervention}

Patients randomised to the intervention will receive 10 $\mathrm{mL}$ of hAF given intravenously as a push dose each day for 5 days. Patients randomised to control will be given $10 \mathrm{~mL}$ of saline placebo intravenously each day for 5 days. hAF and placebo will be produced at CellReGen. CellReGen is unique in the world for being able to process and manufacture hAF for clinical use. ${ }^{19}$ As above, hAF has been approved by the FDA as an HCT/P under 21 CFR $1271^{8}$ for tissue injury.

Patients in both groups will receive standard clinical care for COVID-19, including treatment by a clinical team led by an attending physician. Additional care team members include residents and/or advanced practice clinicians (physician assistants and nurse practitioners), pharmacists, respiratory therapists and nurses. Patients will be managed per standard of care, and in cases of worsening respiratory or haemodynamic status will have their level of care escalated to an intensive care unit (ICU) if indicated.

\section{Blinding}

Intervention (hAF) is naturally near colourless with minimal visual difference compared with saline. To ensure complete blinding, study drug (intervention (hAF) vs placebo) will be prepared in identical containers. Nurses administering the study drug will be blinded to treatment allocation. The participants, treatment team and blinded study coordinators will be blinded to treatment allocation. Objective clinical data recording extraction will be performed by unblinded study staff. Unblinding will happen in cases of data and safety monitoring board (DSMB) concern for patient safety that requires unblinding for treatment.

\section{Primary outcome measure}

The primary outcome is the change in inflammatory biomarkers of CRP from prior to intervention to after the intervention, measured at 6 days.

\section{Secondary outcome measure}

Secondary outcomes and rationale:

1. Safety.

- We will assess adverse events (AE), including the proportion of subjects who experience AEs, and serious adverse events (SAEs) will be summarised descriptively; no inferential tests will be performed.

- Exploratory outcomes for safety include, but are not limited to, the following:

- Whether the patient experienced any postrandomisation AEs while on study (regardless of relatedness to study participation).

- Whether the patient experienced any postrandomisation SAEs while on study.

- Whether the patient experienced any postrandomisation unexpected AEs or SAEs while on study.

2. Death within 30 days.

- Death will be assessed as a plausibly related outcome to decreasing inflammation through hAF.

3. ICU-free days at 30 days.

- ICU-free days will be assessed to quantify the relationship of hAF with critical illness.

4. Hospital length of stay.

- Hospital length of stay will be assessed to quantify the relationship of hAF with critical illness.

5. Need for invasive mechanical ventilation. 
- Need for mechanical ventilation will be assessed to quantify the relationship of inflammation with critical illness.

6. Biomarker levels (IL-6, D-dimer, lactate dehydrogenase).

- To assess the relationship between hAF and inflammatory biomarkers.

7. Need for extracorporeal membrane oxygenation.

- To assess the relationship between hAF and cardiopulmonary failure.

8. Major adverse cardiac events.

- To assess the relationship between hAF and cardiac events.

\section{Ancillary outcome measures}

1. Patient-reported functional status.

- To assess patient's health-related quality-of-life patient-reported outcomes after hAF.

\section{Specimens}

Any additional laboratory specimens (blood), after informed consent, will be processed and stored as required at $-80^{\circ} \mathrm{C}$ according to standard clinical laboratory practices, and adhering to HIPAA regulations for protection of patient identity.

\section{Follow-up}

Table 1 shows the timeline of study procedures and data collection.

\section{Adherence and loss to follow-up}

Study staff will record study drug administration and any missed doses. In cases of patient withdrawal from study, study staff will note this and continue to record clinical and safety outcomes through the duration of data collection. Analysis will occur with intention-to-treat and perprotocol cohorts.

\section{Missing items}

Missing data will be identified by the clinical research coordinator/research assistant (CRC/RA) and sought from the electronic medical record. In cases of persistent missing data, multiple imputation will be used for variables with $<50 \%$ missing.

\section{Sample size}

A sample size of $n=30$ patients per group (total $n=60$ ) is expected, though this may differ slightly because of the randomisation procedure. This is based on results from our pilot study, demonstrating a $>80 \%$ decrease in inflammatory biomarkers among patients who responded to the therapy. If there are 30 subjects in the hAF group and if all subjects in the hAF group complete the study without experiencing an $\mathrm{AE}$, we will be able to state with $95 \%$ confidence that the safety of hAF is at least $90 \%$. Table 2 details the sample size calculation for AEs. If we are unable to enrol 60 patients due to lack of cases, we will expand the study to other hospitals.

\section{Statistical analysis}

This is a trial with subjects randomised to two groups: (1) treatment group with hAF administered intravenously $(\mathrm{n}=30)$, (2) control group with intravenous administration of a saline control $(n=30)$. Both groups will receive standard of care in addition to these treatments.

Descriptive statistics, including mean (SD) and median (IQR), will be used to assess patient characteristics, and clinical and biomarker outcomes. Comparisons may be made stratified on ICU versus floor status at enrolment for variables. Categorical characteristics will be compared using $\chi^{2}$ test or Fisher's exact test. Continuous characteristics will be compared using independent samples t-test and Wilcoxon-Mann-Whitney test. The primary outcome will be compared using analysis of covariance (ANCOVA) and regression. Secondary outcomes will be compared using ANCOVA, Fisher's exact test, $\chi^{2}$ test, t-test or the Wilcoxon-Mann-Whitney test. $95 \%$ CIs and $\mathrm{p}$ values will be reported from all comparisons. Statistical analyses will be conducted in R and STATA, significance will be assessed at the 0.05 level and all tests will be two tailed.

Safety will be assessed by descriptive reporting of the incidence proportion of study-related safety AEs in each study group, with one-sided 95\% binomial exact CIs. No statistical hypothesis test is planned to assess safety, which is consistent even with large phase III trials. Table 2 displays the number of subjects, out of 30 , experiencing no $\mathrm{AE}$ and the corresponding lower bound of the $95 \%$ exact CI.

\section{Safety considerations}

AEs and SAEs will be followed. AEs and SAEs will be defined according to standard practice. AEs will include any untoward medical occurrences associated with the use of hAF, whether or not considered to be intervention related. SAEs, per standard, will be defined as an event that results in death, is life threatening, requires inpatient hospitalisation or prolongs an existing hospitalisation, results in persistent or significant disability or incapacity, results in congenital anomaly/birth defect, or any other event that may jeopardise the subject's health and may require medical or surgical intervention.

For the purposes of this study, events that occur following randomisation through the patient discharge will be reported as AEs. SAEs, unexpected medically attended events and new-onset chronic illnesses will be recorded from randomisation through the day of discharge. Specifically, events that occur following informed consent to participate in the study, but prior to actual randomisation are not AEs. These should be recorded as baseline conditions.

After patient randomisation, all AEs (including SAEs) will be recorded according to relatedness, severity and expectedness, as well as their duration and any treatment prescribed. Any medical condition present at the time of randomisation, recorded in the patient's baseline history at study entry, which remains unchanged or improves (unless the clinician feels it is clinically relevant), will not 

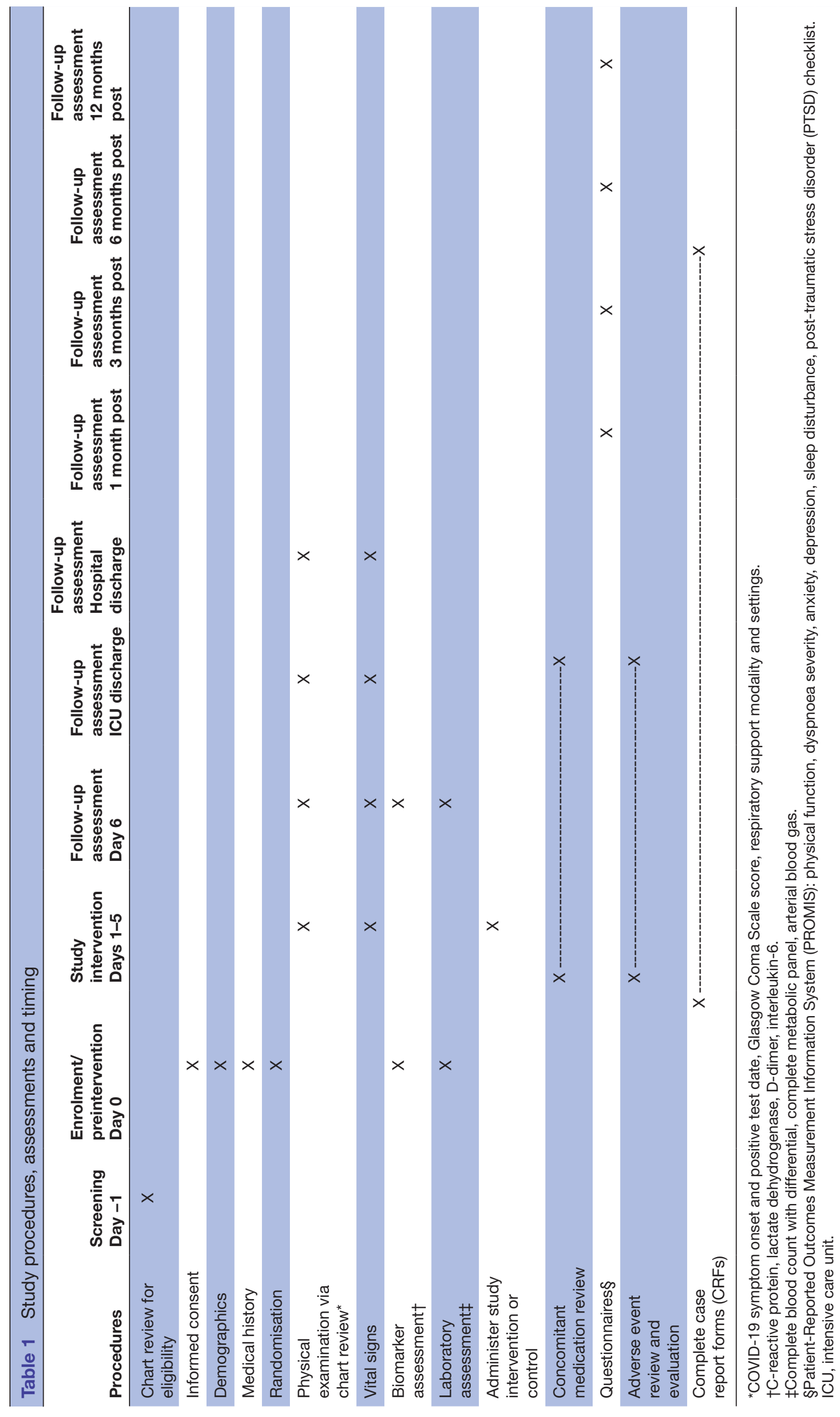

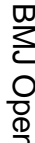

$\stackrel{ }{\vec{*}}$

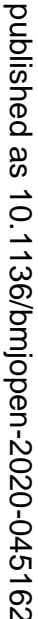

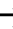

꿍

ఏ్ల

N

뭉 
Table 2 Sample size calculation for adverse events with associated $\mathrm{Cls}$

\begin{tabular}{lll}
\hline $\begin{array}{l}\text { Subjects with no } \\
\text { related adverse } \\
\text { events (n) }\end{array}$ & $\begin{array}{l}\text { Subjects } \\
\text { experiencing a related } \\
\text { adverse event (n) }\end{array}$ & $\begin{array}{l}\text { Lower bound of } \\
\text { the 95\% } \\
\text { exact binomial } \\
\text { Cl for safety }\end{array}$ \\
\hline 30 & 0 & $90 \%$ \\
29 & 1 & $85 \%$ \\
28 & 2 & $80 \%$ \\
27 & 3 & $76 \%$ \\
\hline
\end{tabular}

be recorded as an $\mathrm{AE}$ at subsequent evaluations. However, worsening of a medical condition that was present at the time of randomisation will be considered a new $\mathrm{AE}$ and reported.

\section{Data management and sharing}

All participating investigators and personnel will have obtained Collaborative Institutional Training Initiative and Good Clinical Practice certifications, along with being listed on the site IRB, prior to study involvement. All data or information obtained for the purpose of this trial that could identify participants will be treated as confidential and stored securely.

Patient data collection will be performed at each site by trained and experienced CRCs and RAs. The CRC/ RA will be blinded to treatment allocation. Data will be extracted from the electronic medical record directly into a password-protected web-based server $\left(\right.$ REDCap $\left.^{20}\right)$. All study data are regularly backed up by the University of Utah. Study data will be retained for at least 5 years. Only researchers approved by the IRB and affiliated with the trial will have access to the data. Data quality will be checked for each patient on a regular basis by the study research manager and confirmed by the study principal investigator and site principal investigators. Trial results will be published in peer-reviewed publications on completion of analysis.

\section{Blinded data interpretation}

Data will be analysed by an independent blinded statistician using the unique identifier study ID. Study IDs will be clustered by treatment assignment by an unblinded study investigator, who will label treatment assignment groups as $\mathrm{A}$ and $\mathrm{B}$, corresponding to either intervention (hAF) or placebo. Results will be presented according to treatment group A vs group B, which will be deidentified after analysis is complete.

\section{Patient and public involvement}

No patient or public involvement was present in the planning of the trial.

\section{Data and safety monitoring board}

Safety outcomes will be continuously recorded and reported to a data safety monitoring committee (DSMB). The DSMB will meet prior to first enrolment, and then again to review outcomes after the first patient, then after 20, 40 and 60 patients (end of study). They will meet as needed more often for prespecified SAEs. In the case of safety concern, the DSMB will advise the trial to be stopped by notifying the principal investigator and study team. The DSMB can unblind their review as needed. Additionally, the trial will be internally monitored by an oversight committee consisting of the principal investigator, study coordinator, statistician and data manager. There are no planned interim analyses.

The trial could be terminated in the event the DSMB advises termination for safety reasons or by the principal investigator in case of low recruitment.

\section{Dissemination}

Analysis results from the trial will be published in the peer review literature. Authors of manuscripts will come from the members of the planning committee who fulfil the requirements of the International Committee of Medical Journal Editors (ICMJE). Planning committee members are authors of this manuscript. Additional individuals who fulfil ICMJE requirements may be authors.

\section{DATA SHARING}

To facilitate research reproducibility, replicability, accuracy and transparency, and to comply with ICMJE requirements for clinical trials, ${ }^{21}$ all of the individual participant data collected during the trial, the study protocol, statistical analysis plan, informed consent form, clinical study report and analytic code will be made available indefinitely, following publication, to anyone who wishes to access the data for analysis, on the Open Science Foundation $^{22}$ repository (DOI $10.17605 /$ OSF.IO/WB67N) (https://osf.io/wb67n/) after completion of all analyses, or sooner on reasonable request. Data will be deidentified in accordance with Section 164.514 of the HIPAA.

\section{Patient and public involvement}

Patients or the public were not involved in the design of our research.

\section{SIGNIFICANCE}

As of 9 September 2020, COVID-19 has infected over 27 million people worldwide, of whom $>890000$ have died. ${ }^{23}$ COVID-19 continues to spread, and it is conservatively estimated that it will infect over 16 million Americans. Of infected patients, $15 \%$ require hospitalisation, and 5\%-960 000 Americans-will require intensive care. ${ }^{24}$ Patients with COVID-19 can suffer from profound inflammatory response, which can be associated with hypoxaemia and even cardiopulmonary failure. Many are plagued with cardiac manifestations including myocarditis, accelerated heart failure and arrhythmias. ${ }^{17}$ Lung pathology specimens from the SARS-1 epidemic in 2005 demonstrated diffuse alveolar damage with extensive fibrosis. ${ }^{18}$ Likewise, lung pathology in patients with 
COVID-19 undergoing lung resection for lung cancer (ie, non-autopsy pathology) revealed oedema and patchy, inflammatory cellular infiltrates, in particular with macrophages. ${ }^{19}$ Furthermore, the magnitude of this inflammatory response is correlated with mortality, ${ }^{16}$ and may explain the high mortality in some younger adult patients. Thus, decreasing the inflammatory response during COVID-19 may save lives.

Given the observed ability of hAF to decrease inflammation in various clinical models, the potential for hAF as a treatment for COVID-19 inflammatory syndrome is significant. Our centre is unique in the world for being able to process and manufacture hAF for clinical use. ${ }^{20}$ Our centre has multiple active investigations of hAF for clinical treatment of a variety of conditions, including myocardial ischaemia reperfusion injury, osteoarthritis, wound/burn healing and ocular graft versus host disease. In this randomised clinical trial, we hope to demonstrate the clinical effect of intravenous administration of hAF for patients with moderate COVID-19.

\section{Twitter Joseph E Tonna @JoeTonnaMD}

Contributors JET, JP, NH, GL, JDP and CHS had full access to all the data in the study, take responsibility for the integrity of the data, the accuracy of the data analysis and the integrity of the submission as a whole from inception to published article. JET, JP, NH, GL, JDP and CHS conceived the study design. JET, JP, NH, GL, JDP, AM, CRS, KT and CHS contributed to conduct of the study. JET, JP, NH, GL, JDP, AM, CRS, KT and CHS contributed to data acquisition and analysis. JET, JP, JDP, CRS and CHS drafted the work. All authors revised the article for important intellectual content, had final approval of the work to be published and agree to be accountable for all aspects of the work.

Funding This study was funded by the University of Utah Office of the VicePresident for Research through the Immunology, Inflammation and Infectious Disease (3i) Initiative (No 4-15-20) and the State of Utah CARES Act (№ 8-5-20). JET is supported by a career development award (K23HL141596) from the National Heart, Lung and Blood Institute (NHLBI) of the National Institutes of Health (NIH). This study was also supported, in part, by the University of Utah Study Design and Biostatistics Center, with funding in part from the National Center for Research Resources and the National Center for Advancing Translational Sciences, National Institutes of Health, through grant 5UL1TR001067-02 (formerly 8UL1TR000105 and UL1RR025764).

\section{Competing interests None declared.}

Patient consent for publication Not required.

Provenance and peer review Not commissioned; externally peer reviewed.

Supplemental material This content has been supplied by the author(s). It has not been vetted by BMJ Publishing Group Limited (BMJ) and may not have been peer-reviewed. Any opinions or recommendations discussed are solely those of the author(s) and are not endorsed by BMJ. BMJ disclaims all liability and responsibility arising from any reliance placed on the content. Where the content includes any translated material, BMJ does not warrant the accuracy and reliability of the translations (including but not limited to local regulations, clinical guidelines, terminology, drug names and drug dosages), and is not responsible for any error and/or omissions arising from translation and adaptation or otherwise.

Open access This is an open access article distributed in accordance with the Creative Commons Attribution Non Commercial (CC BY-NC 4.0) license, which permits others to distribute, remix, adapt, build upon this work noncommercially, and license their derivative works on different terms, provided the original work is properly cited, appropriate credit is given, any changes made indicated, and the use is non-commercial. See: http://creativecommons.org/ licenses/by-nc/4.0/.
ORCID iD

Joseph E Tonna http://orcid.org/0000-0001-8879-2628

\section{REFERENCES}

1 Kramer PA, Chacko BK, George DJ, et al. Decreased bioenergetic health index in monocytes isolated from the pericardial fluid and blood of post-operative cardiac surgery patients. Biosci Rep 2015;35:BSR20150161. doi:10.1042/BSR20150161

2 Sirch J, Ledwon M, Püski T, et al. Active clearance of chest drainage catheters reduces retained blood. J Thorac Cardiovasc Surg 2016;151:832-8.

3 JW D. Skin transplantation: with a review of 550 cases at the Johns Hopkins Hospital. Johns Hopkins Med J 1910;15:307.

4 Cargnoni A, Di Marcello M, Campagnol M, et al. Amniotic membrane patching promotes ischemic rat heart repair. Cell Transplant 2009;18:1147-59.

$5 \mathrm{Kim} \mathrm{HG}$, Choi OH. Neovascularization in a mouse model via stem cells derived from human fetal amniotic membranes. Heart Vessels 2011;26:196-205.

6 Food and Drug Administration (FDA). Code of federal regulations, title 21, part 1271. Available: https://www.ecfr.gov/cgi-bin/text-idx? $\mathrm{SID}=567 \mathrm{~d} 39158 \mathrm{cc} 375 \mathrm{~b} 792 \mathrm{efedc} 7 \mathrm{fe} 07689 \mathrm{~b} \& \mathrm{mc}=$ true\&node=pt21.8. 1271\&rgn=div5 [Accessed 22 Jun 2020].

7 Marsh KM, Ferng AS, Pilikian T, et al. Anti-Inflammatory properties of amniotic membrane patch following pericardiectomy for constrictive pericarditis. J Cardiothorac Surg 2017;12:6.

8 Pierce J, Jacobson P, Benedetti E, et al. Collection and characterization of amniotic fluid from scheduled C-section deliveries. Cell Tissue Bank 2016;17:413-25.

9 Mohammadi AA, Seyed Jafari SM, Kiasat M, et al. Effect of fresh human amniotic membrane dressing on graft take in patients with chronic burn wounds compared with conventional methods. Burns 2013;39:349-53.

10 Hao Y, Ma DH, Hwang DG, et al. Identification of antiangiogenic and antiinflammatory proteins in human amniotic membrane. Cornea 2000;19:348-52.

11 Koob TJ, Rennert R, Zabek N, et al. Biological properties of dehydrated human amnion/chorion composite graft: implications for chronic wound healing. Int Wound J 2013;10:493-500.

12 Brandt FT, Albuquerque CD, Lorenzato FR. Female urethral reconstruction with amnion grafts. Int J Surg Investig 2000;1:409-14.

13 Burman S, Tejwani S, Vemuganti GK, et al. Ophthalmic applications of preserved human amniotic membrane: a review of current indications. Cell Tissue Bank 2004;5:161-75.

14 Liu B, Li M, Zhou Z, et al. Can we use interleukin-6 (IL-6) blockade for coronavirus disease 2019 (COVID-19)-induced cytokine release syndrome (CRS)? J Autoimmun 2020;111:102452.

15 Chen J, Qi T, Liu L, et al. Clinical progression of patients with COVID-19 in Shanghai, China. J Infect 2020;80:e1-6.

16 Yang Y, Shen C, Li J. Exuberant elevation of IP-10, MCP-3 and IL-1ra during SARS-CoV-2 infection is associated with disease severity and fatal outcome 2020 .

17 Conti P, Ronconi G, Caraffa A, et al. Induction of pro-inflammatory cytokines (IL-1 and IL-6) and lung inflammation by Coronavirus-19 (COVI-19 or SARS-CoV-2): anti-inflammatory strategies. J Biol Regul Homeost Agents 2020;34:327-331.

18 Chan A-W, Tetzlaff JM, Altman DG, et al. Spirit 2013 statement: defining standard protocol items for clinical trials. Ann Intern Med 2013;158:200-7.

19 Mao Y, Pierce J, Singh-Varma A, et al. Processed human amniotic fluid retains its antibacterial activity. J Trans/ Med 2019;17:68.

20 Harris PA, Taylor R, Thielke R, et al. Research electronic data capture (REDCap)--a metadata-driven methodology and workflow process for providing translational research informatics support. J Biomed Inform 2009;42:377-81.

21 Taichman DB, Sahni P, Pinborg A, et al. Data Sharing Statements for Clinical Trials - A Requirement of the International Committee of Medical Journal Editors. N Engl J Med 2017;376:2277-9.

22 Foster ED, Deardorff A. Open science framework (OSF). J Med Libr Assoc 2017;105:203-6.

23 World Health Organization. Weekly operational update on COVID-19-9 September 2020. Available: https://www.who.int/docs/ default-source/coronaviruse/weekly-updates/wou-9-september2020-cleared.pdf?sfvrsn=d39784f7_2 [Accessed 10 Sep 2020].

24 Emanuel EJ, Persad G, Upshur R. Fair allocation of scarce medical resources in the time of Covid-19. N Engl J Med 2020. 